\title{
Elastic Stable Intra-Medullary Nailing (ESIN) in Management of Fracture Clavicle
}

\author{
Sherin A Khalil, Osama M Essawy, Mina S Boktor
}

Department of Orthopedic, Benha faculty of medicine, Benha University, Egypt.

Correspondence to: Mina

S Boktor, Department of Orthopedic, Benha faculty of medicine, Benha University, Egypt.

Email:

dr.minaboktor@gmail.com

Received: 11 October 2019

Accepted: 11 May 2021

\begin{abstract}
:
Back ground: Clavicle fractures accounting for 2.6 to $10 \%$ of all adult fractures are usually treated non-operatively. There is an increasing trend toward their surgical fixation. The aim of our study was to investigate the outcome following titanium elastic stable intramedullary nailing (ESIN) for mid shaft clavicular fractures. Materials and Methods: A total of 20 patients, which met inclusion criteria, were reviewed retrospectively. There were 14 males and 6 females. The mean age was 37.5 years. $60 \%$ of the patients were poly-traumatized. The patients were assessed for clinical/radiological union and by Oxford Shoulder and QuickDASH scores. 40\% patients required open reduction. Results: Ninety $\%$ union was achieved at average of 11.3 weeks. The average follow-up was 6 months. The average Oxford Shoulder and QuickDASH scores were 43.5.6 and 8.5, respectively. No cases of nail migration were reported. Conclusion: In our hands, ESIN is safe and minimally invasive with good patient satisfaction, cosmetic appearance, and early return to daily activities. Considering cosmesis the ESIN has much more superior
\end{abstract} outcome when compared to fixation by plate and screws.

Key words: clavicle, intramedullary, ESIN, Nancy nail

\section{Introduction:}

The clavicle is one of the most frequently broken bones in the human body. It also serves as an important and easily located bony landmark due to its superficial location and projection from the trunk. This long bone has a number of different features, it has no medullary cavity, and it is the first to ossify in the fetus (5th-6th week). Despite being a long bone, it develops in membrane and not cartilaginous ossification, it is the most common long bone liable to be broken in the body (1). 
Clavicle fractures are among the most common skeletal injuries accounting for 2.6 to $10 \%$ of all adult fractures. Historically, non-operative treatment of midshaft clavicular fractures was considered the gold standard of care. Recent evidence, however, reveals that the final result of non-surgically midshaft clavicular fractures, particularly those with quite large displacements or shortening, is not like that which was previously thought. The increasing popularity and rate of surgical fixations in recent years has challenged the nonoperative treatment (2).

Mid-shaft fractures of the clavicle are considered the most common form of clavicle fractures and about half of them are displaced fractures. They have been managed non-operatively over the years. However, severe displacement and comminution of these fractures have warranted the requirement of operative intervention, due to increased incidence of mal-union, worsening of shoulder functions etc., after conservative management. The introduction of locking compression plates, have increased the incidence of operative intervention in the management of these injuries (3).

Elastic stable intra-medullary nails (ESIN) are biological minimally invasive method of fixation of fractures which aims to achieve level of reduction and appropriate stabilization. Stabilization is achieved with flexible intramedullary nails that have been pre-contoured to provide some elastic properties. This enables them to provide sufficient stability to permit early movement (3).

The aim of this work is to evaluate the functional outcome and to assess the anatomical restoration of the clavicle with radiographic measurements after intramedullary fixation of clavicular fractures by elastic stable intra-medullary nailing (ESIN)

\section{Patient and method}

A prospective study on 20 patients operated upon at Department of Orthopaedic Surgery Benha university Hospital. This research was done between September 2017 and September 2019, written consents were taken from the patients operated upon

All patients suffered from displaced clavicular fractures managed by elastic stable intra-medullary nailing (ESIN), and followed up for a minimum period of 3 months, the maximum period of follow up was 6 months. Patients were evaluated preoperatively by history taking, physical examination, imaging, laboratory investigation and consent taking 
Clavicular fractures operated upon according to Allman' classification, mainly type I, also II and III fractures will be included both genders were. Patients with $\mathrm{AC}$ joint dislocation, fractures involving articular surface, open fractures, old fractures more than 3 weeks and revision cases post failed open reduction and intramedullary fixation by plate and screws were excluded. Active range of movement exercises of the shoulder will be commenced on post-operative days 18-20. Stitches were removed 10-14 days post-operative. Second follow-up at 6 weeks for plain radiographic evaluation of fracture healing.

The patients were evaluated at $3,4,6$ months follow-up. Standard radiographs of the affected. They will be evaluated for union and loss of reduction

The patients were evaluated radiologically according to union rate and time and clinically according to range of motion (ROM) and numeric rating scale for pain (NPRS).

QuickDASH shoulder score, which is a clinician-based outcomes measure, was the scoring system used. QuickDASH score is scaled from 0 to 55 , with higher scores indicating a greater level of disability.

\section{Results}

This study was conducted on 20 patients with following result

Study shows that $70 \%$ of patients were males and $30 \%$ females with mean age 37.5 years

Thirty per cent of patients had recessive correction. $60 \%$ of patients were poly traumatized and $40 \%$ isolated. The mean Quick Dash Score was 8.6 and mean Oxford Score was 41.8

Ninety per cent of patients had union and only $10 \%$ were non-union. Intra-operatively $60 \%$ of patients had closed reduction and $40 \%$ needed open reduction. The mean time to union was 13.4 months with min-max 1016 months. As regard mean flexion, extension, abduction, external rotation and adduction were 148, 44.5, 174, 94 and 111 respectively. Mean size of nail was 2.8. Mean operative time was 32.4 minutes with $\min -\max 27-40$ 


\begin{tabular}{llll}
\hline & Min. - Max. & Mean \pm SD & Median \\
\hline Time to union(months) & $10.0-16.0$ & $13.40 \pm 2.23$ & 13.0 \\
Flexion & $130.0-160.0$ & $148.0 \pm 12.81$ & 150.0 \\
Extension & $30.0-50.0$ & $44.50 \pm 5.36$ & 45.0 \\
Abduction & $160.0-180.0$ & $174.0 \pm 9.40$ & 180.0 \\
\hline
\end{tabular}

\section{Statistical method}

Data were statistically described in terms of mean \pm standard deviation $( \pm \mathrm{SD})$, median and range, or frequencies (number of cases) and percentages when appropriate. Comparison of numerical variables between the study groups will be done using Student $\mathrm{t}$ test for independent samples. For comparing categorical data, Chi square $(\chi 2)$ test will be performed. Exact test will be used instead when the expected frequency is less than 5. Correlation between various variables will be done using Pearson moment correlation equation. $\mathrm{p}$ values less than 0.05 will considered statistically significant. All statistical calculations will be done using computer programs SPSS (Statistical Package for the Social Science; SPSS Inc., Chicago, IL, USA) version 15 for Microsoft Windows.

\section{Discussion}

Fracture of the clavicle accounts for about $4 \%$ of all fractures and $35 \%$ of shoulder girdle injuries. Around $80 \%$ of fractures of the clavicle involve the midshaft and over half of these fractures are displaced. Traditionally, clavicular fractures have been treated non-operatively, even with substantial displacement. (4)

In athletic patient especially due to the poor outcome of DMCF treated conservatively and with increasing patients' expectations, operative treatment of DMCF becomes more and more popular to improve post fracture life style and early return to work and early return to practice sports (5).

Typical complications of plate fixation include infection, hypertrophic scars, implant loosening and re-fracture after implant removal with an incidence of up to 23\%. Furthermore, due to the implant design, patients report skin irritation and hardware prominence causing unnecessary pain (6).

In the current study we aimed to evaluate the functional outcome and to assess the anatomical restoration of the clavicle with radiographic measurements after intramedullary fixation of clavicular fractures by elastic stable intra-medullary nailing 
(ESIN). Surgical time is considerable whether there was a need for open reduction or not (7).

In comparison with other studies done discussing the effectiveness of ESIN in treatment of clavicular fractures, our study 20 patients were included with mean age 37.5 years, $70 \%$ of patients were males and $30 \%$ females and $30 \%$ had recessive predominance In another study (9), 46 patients were included divided into 2 groups according to the comminution in the fracture site where group 1 has no comminution and group 2 has comminution at the fracture site. The average patients' age at the time of operation was $36 \pm 11$ years for group I versus $39 \pm 9$ years for group II with female predominance and an average $44 \%$ recessive predominance (8)

In another study (10) a study was made on 38 patients; $60 \%$ male predominance and $30 \%$ recessive hand while in another a study was made on 36 patients; $55 \%$ male predominance and $28 \%$ had the injury in the recessive hand while another study performed on 33 patients; $60 \%$ male predominance and $40 \%$ had recessive hand.(9)

In the current study we found that the mean Quick Dash Score was (8.6) and mean Oxford Score was (41.8) Those results were near to another study (10) that discussed ESIN in mid shaft clavicular fractures as they showed that the average QuickDASH score (6.7). The average was (range, 0-13.6) and Oxford Shoulder score was 45.6 (range, 37-48) at the last follow-up .While the mean DASH scores in another study (12) were

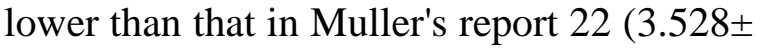
1.567 vs $5 \pm 2.3$ ) while the quick dash score and the oxford score in another study (11) was 5 (range,0-11), 44(range 36-44) (10). There was no significant difference comparing patients who were treated with an open versus a closed technique. If appropriately indicated we believe that using ESIN is an adequate and successful operative technique for DMCF. There were no significant differences in shoulder function after either procedure (11).

\section{Conclusion}

ESIN is a safe and minimally-invasive technique when appropriately indicated of DMCF - even when additional open reduction is needed. Good cosmetic and functional results are achieved while less operative time and fluoroscopy time are used. ESIN is a promising alternative to plate fixation. 


\section{References:}

1. Dugas, J.R, O'Brien KBT, S.J., William G.E., and S.P. Kevin. 2000. "Anatomy of the shoulder, in Principles and practice of Orthopaedic sports medicine." Lippincott Williams and Wilkins 1st edition: 329-378.

2. Robinson, CM, and DA Cairns. 2004. "Primary nonoperative treatment of displaced lateral fractures of the clavicle." Bone Joint Surg Am 778-82.

3. Robinson, CM1, CM Court-Brown, MM McQueen, and Wakefield AE. 2004. "Estimating the risk of nonunion following nonoperative treatment of a clavicular." J Bone Joint Surg Am 1359-65.

4. Waldmann, S1, E1 Benninger, Meier, and C1. 2018. "Nonoperative Treatment of Midshaft Clavicle Fractures in Adults." Open Orthop $J$ $12: 1-6$.

5. Kujath, P. 2010. "Complicated skin, skin structure and soft tissue infections - are we threatened by multi-resistant pathogens?" European Journal of Medical Research 15:544553.

6. Kadakia, AP, R Rambani, F Qamar, and et al. 2012. "Titanium elastic stable intramedullary nailing of displaced midshaft clavicle fractures: A review of 38 cases." nt $J$ Shoulder Surg 6(3):82-85.

7. Rokito AS, Zckerman JD. 2002. "A comparison of nonoperative and operative treatment of type
II distal clavicle." Bull Hosp Jt Dis 61(1-2): 329.

8. Xie, L1, Z1 Zhao, S1 Zhang, and Y2 Hu. 2018. "Intramedullary fixation versus plate fixation for displaced mid-shaft clavicle fractures: A systematic review of overlapping metaanalyses." Medicine (Baltimore).

9. Yasser, RA, MR Ali, BS Walid, and et al. 2017. "Outcome of elastic stable intramedullary nailing of displaced midshaft clavicular fracture: does the presence of fracture comminution differ?" The Egyptian Orthopaedic Journal 52:18-25.

10. Kadakia, AP, R Rambani, F Qamar, and et al. 2012. "Titanium elastic stable intramedullary nailing of displaced midshaft clavicle fractures: A review of 38 cases." nt $J$ Shoulder Surg 6(3):82-85.

11.KF, Braun, Siebenlist S, and Sandmann GH. 2014. "Functional results following titanium elastic-stable intramedullary nailing (ESIN) of mid-shaft." Acta Chir Orthop Traumatol Cech $118-21$.

12.Braun, KF, S Siebenlist, Sandmann, and GH. 2014. "Functional results following titanium elastic-stable intramedullary nailing (ESIN) of mid-shaft clavicle fractures." Acta Chir Orthop Traumatol Cech 81(2):118-21.

To cite this article: Sherin A Khalil, Osama M Essawy, Mina S Boktor. Elastic Stable IntraMedullary Nailing (ESIN) in Management of Fracture Clavicle. BMFJ 2021;38 (orthopedic surgery): 60-65. DOI: 10.21608/bmfj.2021.18049.1094 